\title{
Delay-induced synchronization phenomena in an array of globally coupled logistic maps
}

\author{
A. C. Martí and C. Masoller \\ Instituto de Física, Facultad de Ciencias, Universidad de la Republica, Iguá 4225, 11400 Montevideo, Uruguay
}

(Received 6 December 2002; published 27 May 2003)

\begin{abstract}
We study the synchronization of a linear array of globally coupled identical logistic maps. We consider a time-delayed coupling that takes into account the finite velocity of propagation of the interactions. We find globally synchronized states in which the elements of the array evolve along a periodic orbit of the uncoupled map, while the spatial correlation along the array is such that an individual map sees all other maps in his present, current, state. For values of the nonlinear parameter such that the uncoupled maps are chaotic, time-delayed mutual coupling suppresses the chaotic behavior by stabilizing a periodic orbit that is unstable for the uncoupled maps. The stability analysis of the synchronized state allows us to calculate the range of the coupling strength in which global synchronization can be obtained.
\end{abstract}

DOI: 10.1103/PhysRevE.67.056219

PACS number(s): 05.45.Xt, 05.65.+b, 05.45.Ra

\section{INTRODUCTION}

Coupled oscillator models are widely used to model complex dynamics in nonequilibrium extended systems, and their synchronization has attracted a lot of attention in recent years [1]. In studies of coupled ensembles of nonlinear oscillators, different situations have been considered (identical or nonidentical units, periodic or chaotic single-unit behavior, local or global coupling), and a rich variety of synchronization phenomena has been found (for a recent review, see Ref. [2]).

In the field of coupled map lattices, the paradigmatic model, originally introduced by Kaneko [3,4], is the ensemble of $N$ logistic maps with mean field global coupling:

$$
x_{i}(t+1)=(1-\epsilon) f\left[x_{i}(t)\right]+\frac{\epsilon}{N} \sum_{j=1}^{N} f\left[x_{j}(t)\right],
$$

$i \in[1, N], f(x)=\operatorname{ax}(1-x)$, and $\epsilon$ is the coupling strength. For relatively large coupling, global (full) synchronization occurs: the array synchronizes on the manifold $x_{1}=\ldots$ $=x_{N}$, where the dynamics of an element is generated by the uncoupled map. For weaker coupling, cluster (or partial) synchronization occurs: the array splits into $K$ clusters of $N_{1}, \ldots, N_{K}$ elements mutually synchronized $[5,6]$.

A characteristic of many biological and physical systems is time-delayed coupling in the interaction among many units. Two different situations can be distinguished: when the retardation time in the coupling is the same for all the units, and when the retardation time is different for the different units. An example of the first case was studied in Refs. [7,8], which considered an array of diode lasers with delayed coupling via an external reflector. The retardation time in the coupling is the same for all lasers since it given by the external cavity round-trip time. The delay time was found to induce in-phase synchronization of the array; a behavior that was interpreted in terms of generalized Kuramoto phase equations.

Globally coupled logistic maps with time-delay interactions were studied in Ref. [9]; the maps were coupled with the same delay $k$ :

$$
x_{i}(t+1)=(1-\epsilon) f\left[x_{i}(t)\right]+\frac{\boldsymbol{\epsilon}}{N} \sum_{j=1}^{N} f\left[x_{j}(t-k)\right] .
$$

The dynamics of the array was found to be strongly sensitive to the value of the delay time, which can increase the probability of the cluster state for small coupling strengths, and can also break up the cluster state for large coupling. Suppression of spatiotemporal chaos in a linear array with local (nearest neighbor) coupling, via local and global timedelayed feedback was demonstrated numerically and analytically in Refs. [10,11].

In the case of globally coupled units, the introduction of distance-dependent time delays makes the spatial coordinates of an element relevant in spite of the infinite range of the mean-field interaction. This situation was considered in Ref. [12] for one-dimensional arrays of coupled phase oscillators. It was shown that in the limit of short delays, the ensemble approaches a state of frequency synchronization, and that this state might develop a spatial nontrivial distribution of phases. In two-dimensional arrays, distance-dependent time delays induce a variety of patters including traveling rolls, steady patterns, spirals, and targets [13].

Here we study the effects of distance-dependent retarded coupling in a linear array of logistic maps:

$$
x_{i}(t+1)=(1-\epsilon) f\left[x_{i}(t)\right]+\frac{\epsilon}{N} \sum_{j=1}^{N} f\left[x_{j}\left(t-\tau_{i j}\right)\right],
$$

where $\tau_{i j}=k|i-j|$ is proportional to the distance between the $i$ th and $j$ th maps and $k$ is the inverse of the velocity of the signal that travels through the array. In a previous work [14], we considered the case in which the uncoupled maps evolve in a periodic orbit of period 2 (when $3 \leqslant a \leqslant 1$ $+\sqrt{6})$. We found that for weak coupling the array divides into clusters, and the behavior of the individual elements within each cluster depend on the delay times. For strong enough coupling global synchronization occurs, where the dynamics of an element is periodic of period 2, generated by the uncoupled logistic map. The spatial correlation of the elements along the array is such that if $k$ is even, at time $t$ all elements are in the same state, while if $k$ is odd, at time $t$ 
neighboring elements are in different states. In both cases an individual map sees all other maps in its present, current, state.

In this paper we extend the previous study and consider that the uncoupled maps can be either periodic or chaotic (i.e., $3 \leqslant a \leqslant 4$ ). We find that for adequate coupling strength and time delay, global synchronization occurs. In the globally synchronized state all elements evolve along a periodic orbit of the uncoupled logistic map. Remarkably, this orbit might be unstable for the uncoupled maps. In particular, when the uncoupled maps are chaotic, time-delayed coupling might suppress chaos, stabilizing an unstable periodic orbit. For small arrays we study the stability of the globally synchronized solution and calculate the minimum coupling strength above which the unstable orbit of the uncoupled maps becomes stable for the time-delayed coupled maps. The numerical simulations are in excellent agreement with the stability analysis.

This paper is organized as follows. In Sec. II we analyze the existence and the stability of the globally synchronized state. In Sec. III we present results of the numerical simulations and the stability analysis. Finally, in Sec. IV we present a summary and the conclusions.

\section{GLOBALLY SYNCHRONIZED SOLUTIONS}

A special class of solutions of Eq. (3) is characterized by the fact that, for all pairs $i, j$, the signal received by map $i$ at each time corresponds to a delayed state of map $j$, which coincides with the present state of map $i$ :

$$
x_{j}\left(t-\tau_{i j}\right)=x_{i}(t) .
$$

Thus, each element "perceives" the array as being fully synchronized, in spite of the fact that the simultaneous states of different elements might not coincide. In these globally synchronized solutions, each element evolves along a limit cycle of period $P$ of the uncoupled logistic map with a given phase, such that we can write

$$
x_{i}(t)=x_{0}\left(t+\phi_{i}\right)
$$

with $x_{0}(t)$ a particular realization of the limit cycle, used as a reference orbit. The condition for this solution to satisfy the evolution equation is

$$
\phi_{i}-\phi_{j}+m_{i j} P=\tau_{i j}=k|i-j|
$$

for all $i$ and $j$, where $m_{i j}$ are arbitrary integer numbers. The symmetry of the delays, $\tau_{i j}=\tau_{j i}$, implies that

$$
\phi_{i}-\phi_{j}+m_{i j} P=\phi_{j}-\phi_{i}+m_{j i} P \text {. }
$$

Thus, the phase differences $\phi_{i}-\phi_{j}$ cannot be arbitrary, but have to be either $\phi_{i}-\phi_{j}=n_{i j} P$ or $\phi_{i}-\phi_{j}=P / 2+n_{i j} P$, with $n_{i j}$ an integer number.

We shall refer to solutions with $\bmod \left(\phi_{i}-\phi_{j}, P\right)=0 \forall i$ and $j$ as in-phase solutions, and solutions with $\bmod \left(\phi_{i+1}\right.$ $\left.-\phi_{i}, P\right)=P / 2 \forall i$ as antiphase solutions. Since $\bmod \left(\phi_{i+1}\right.$ $\left.-\phi_{i}, P\right)$ is an integer number, the period $P$ of the orbit for the antiphase solution has to be even. The in-phase and antiphase solutions verify Eq. (6) only for certain delay times. For the in-phase solution,

$$
\bmod \left(\phi_{i}-\phi_{j}, P\right)=\bmod (k|i-j|, P)=0
$$

$\forall i$ and $j$ only if $k=n P$, with $n$ an integer number; for the antiphase solution,

$$
\bmod \left(\phi_{i+1}-\phi_{i}, P\right)=\bmod (k, P)=P / 2
$$

only if $k=P / 2+n P$, with $n$ an integer number.

The existence of these globally synchronized states is independent of the coupling strength; the only requirement is that the periodic orbit is a solution (stable or unstable) of the logistic map.

To analyze the stability of the globally synchronized solutions, we turn the delayed equation (3) into a nondelayed equation by the introduction of auxiliary variables:

$$
y_{i m}(t)=x_{i}(t-m)
$$

where $1 \leqslant i \leqslant N$ and $0 \leqslant m \leqslant M$ with $M=\max \left(\tau_{i j}\right)$. In terms of these new variables, Eq. (3) becomes

$$
\begin{aligned}
& y_{i m}(t+1) \\
& =\left\{\begin{array}{l}
y_{i, m-1}(t), \text { if } m \neq 0, \\
(1-\epsilon) f\left[y_{i 0}(t)\right]+\frac{\epsilon}{N} \sum_{j=1}^{N} f\left[y_{j, k|j-i|}\right], \quad \text { if } m=0 .
\end{array}\right.
\end{aligned}
$$

Next we define the vector

$$
\begin{aligned}
Z= & \left(y_{10}, y_{20}, \ldots, y_{N 0} ; y_{11}, y_{21}, \ldots, y_{N 1} ; \ldots ;\right. \\
& \left.\times y_{1 M}, y_{2 M}, \ldots, y_{N M}\right),
\end{aligned}
$$

which has $N(M+1)$ components. The antiphase solutions of period 2 can be written as

$$
\begin{aligned}
& Z_{A}^{1}=\left(x_{a}, x_{b}, \ldots ; x_{b}, x_{a}, \ldots\right), \\
& Z_{A}^{2}=\left(x_{b}, x_{a}, \ldots ; x_{a}, x_{b}, \ldots\right),
\end{aligned}
$$

and the in-phase solutions of period 2 as

$$
\begin{aligned}
& Z_{I}^{1}=\left(x_{a}, x_{a}, \ldots ; x_{b}, x_{b}, \ldots\right), \\
& Z_{I}^{2}=\left(x_{b}, x_{b}, \ldots ; x_{a}, x_{a}, \ldots\right),
\end{aligned}
$$

where $x_{a}$ and $x_{b}$ are the points of the period 2 orbit of the logistic map. We rewrite Eq. (11) as

$$
z_{i}(t+1)=F_{i}\left[z_{1}(t), \ldots, z_{N(M+1)}(t)\right] .
$$

The in-phase and antiphase solutions are fixed points of $F^{2}$ :

$$
F\left(F\left(Z_{I, A}^{1,2}\right)\right)=F\left(Z_{I, A}^{2,1}\right)=Z_{I, A}^{1,2} .
$$


To analyze the stability of these solutions, we need to calculate the eigenvalues of the $N(M+1) \times N(M+1)$ matrix A of components

$$
A_{i j}=\left.\left.\frac{\partial F_{i}}{\partial z_{k}}\right|_{Z=Z_{I, A}^{2}} \frac{\partial F_{k}}{\partial z_{j}}\right|_{Z=Z_{I, A}^{1}} .
$$

We observe that the matrix $\mathbf{A}$ is the product of two $N(M$ $+1) \times N(M+1)$ matrices, $\mathbf{A}=\mathbf{M}\left(Z_{I, A}^{2}\right) \times \mathbf{M}\left(Z_{I, A}^{1}\right)$, where

$$
M_{i j}\left(Z_{I, A}^{1,2}\right)=\left.\frac{\partial F_{i}}{\partial z_{j}}\right|_{Z=Z_{I, A}^{1,2}}
$$

The matrix $\mathbf{M}$ can be cast as a set of $M^{2}$ blocks of dimension $N \times N$. Denoting these blocks as $\mathcal{F}_{i j}$, with $i, j=0, \ldots, M$, we have

$$
\mathbf{M}=\left(\begin{array}{cccc}
\mathcal{F}_{00} & \mathcal{F}_{01} & \ldots & \mathcal{F}_{0 M} \\
\mathcal{F}_{10} & \mathcal{F}_{11} & \cdots & \mathcal{F}_{1 M} \\
\vdots & & & \vdots \\
\mathcal{F}_{M 0} & \mathcal{F}_{M 1} & \cdots & \mathcal{F}_{M M}
\end{array}\right)
$$

In the case of antiphase solution, using Eq. (11) is easy to see that

$$
\mathcal{F}_{00}=\left(\begin{array}{cccc}
{[1-(N-1) \epsilon / N] f^{\prime}\left(x_{a}\right)} & 0 & \cdots & 0 \\
0 & {[1-(N-1) \epsilon / N] f^{\prime}\left(x_{b}\right)} & \cdots & 0 \\
\vdots & 0 & \cdots & {[1-(N-1) \epsilon / N] f^{\prime}\left(x_{b}\right)}
\end{array}\right) .
$$

The blocks $\mathcal{F}_{0 j}$ are nondiagonal matrices; for example,

$$
\mathcal{F}_{01}=\left(\begin{array}{ccccc}
0 & \epsilon / N f^{\prime}\left(x_{a}\right) & 0 & \ldots & 0 \\
\epsilon / N f^{\prime}\left(x_{b}\right) & 0 & \epsilon / N f^{\prime}\left(x_{b}\right) & \ldots & 0 \\
0 & \epsilon / N f^{\prime}\left(x_{a}\right) & 0 & \ldots & 0 \\
\vdots & & & & \vdots \\
0 & 0 & \ldots & 0 & \epsilon / N f^{\prime}\left(x_{a}\right) \\
0 & 0 & \ldots & \epsilon / N f^{\prime}\left(x_{b}\right) & 0
\end{array}\right) .
$$

The blocks $\mathcal{F}_{i j}$ with $i>0$ have all components equal to 0 , except the blocks $\mathcal{F}_{i+1, i}$ which are $N \times N$ identity matrices.

In spite of the fact that the Jacobian matrix $\mathbf{A}$ appears to have a great deal of structure, it does not present a clear symmetry. We did not find any similarity transformation that facilitated the diagonalization of the matrix. Thus, the eigenvalues had to be calculated numerically.

\section{RESULTS}

In this section we present numerical simulations and results of the stability analysis, which demonstrate global synchronization in the in-phase and antiphase solutions discussed in the previous section. The stability analysis can only be done for small arrays and small delay times, since the size of the matrix $A[\mathrm{Eq} .(17)]$ increases as $k N^{2}$. For large arrays and/or large delays, we simulate Eq. (3). To solve delay equation (3), we need to specify the evolution of $x_{i}(t)$ at times $1 \leqslant t \leqslant \max \left(\tau_{i j}\right)$. We evaluated this by taking for $x_{i}(1)$ a random number ranging from 0 to 1 and by letting the array evolve initially without coupling.

First, we show results for the antiphase solution, which exists for $k$ even. For $k=1$, we find that for all values of $a$ there is a value of $\epsilon$ above which the antiphase solution of period 2 is stable. Figure 1 shows the absolute value of the maximum eigenvalue, $\left|\lambda_{\max }\right|$, as a function of $\epsilon$, for an array of $N=12$ maps and three different values of the parameter $a$. For $a=3.5$ (dot-dashed line), the maps without coupling evolve in a limit cycle of period 4; for $a=3.83$ (dashed line), the maps without coupling evolve in a limit cycle of period 3 ; and for $a=4$ (solid line), the maps without coupling are chaotic. For clarity, the dotted line indicates the stability boundary $\left|\lambda_{\max }\right|=1$. In the three cases, for large enough coupling the antiphase solution of period 2 is stable $\left(\left|\lambda_{\max }\right|<1\right)$. Notice that the coupling strength above which the solution is stable increases with increasing $a$.

We verified numerically that for larger arrays, the antiphase solution is stable. Figure 2(a) displays, as an example, a bifurcation diagram for $N=50(a=4$ and $k=1)$. The bifurcation diagram is done in the following way: we chose the same initial condition for all values of $\epsilon$, and we plot the 100 time-consecutive values $x_{i}(t)$ (with $t$ large enough) for a given element $i$ of the array. Figure 2(b) displays the same but for a neighboring element. Above a certain coupling strength, the array synchronizes in the period-2 orbit of the uncoupled map, and the bifurcation diagram for the two elements coincide. The synchronization in the period- 2 orbit is surprising since for $a=4$, the period- 2 orbit 


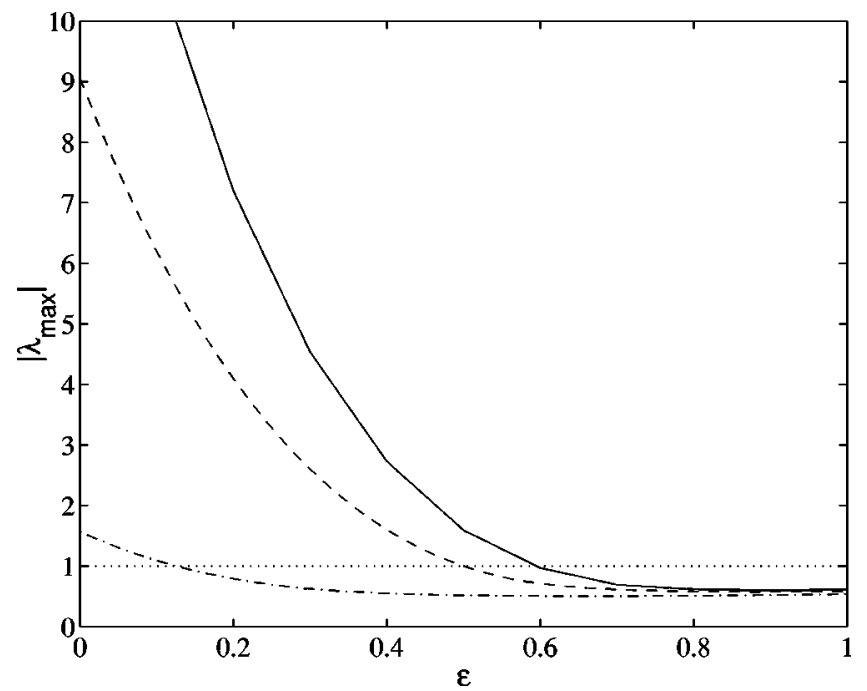

FIG. 1. Stability analysis of the antiphase solution of period 2 for $k=1$ and $N=12$. We plot the largest eigenvalue of the matrix $A$ [Eq. (17)] vs the coupling strength for $a=3.5$ (dot-dashed line), $a$ $=3.83$ (dashed line), and $a=4$ (solid line).

is unstable for the uncoupled maps. While the antiphase solution is stable for $\epsilon \geqslant 0.6$ (see Fig. 1), Fig. 2 shows that the array synchronizes in this solution for a slightly larger coupling strength $(\epsilon \sim 0.7)$. The critical coupling strength $\epsilon_{\text {crit }}$, above which global synchronization occurs, depends slightly on the initial condition, and increases with increasing $a$ and $N$. Figure 3 displays the critical value of $\epsilon$ (calculated averaging over 100 different initial conditions) vs $a . \epsilon_{c r i t}$ increases linearly in the parameter region where the uncoupled maps are periodic, and abruptly in the parameter region where the uncoupled maps are chaotic. Figure 4 (solid line) shows that $\epsilon_{c r i t}$ also increases with increasing system size $N$.

Notice that below the critical coupling strength $\epsilon_{c r i t}$, the bifurcation diagrams shown in Figs. 2(a) and 2(b) differ. This is due to the fact that for $\epsilon<\epsilon_{c r i t}$, the array splits into a complex clustered structure. The clustering behavior in the simpler case where the uncoupled maps evolve in a period-2 orbit was studied in Ref. [14].

For larger time delays and $k$ odd, the interval of coupling strength in which the antiphase solution of period 2 is stable becomes more narrow. As an example, Fig. 5 displays $\left|\lambda_{\max }\right|$
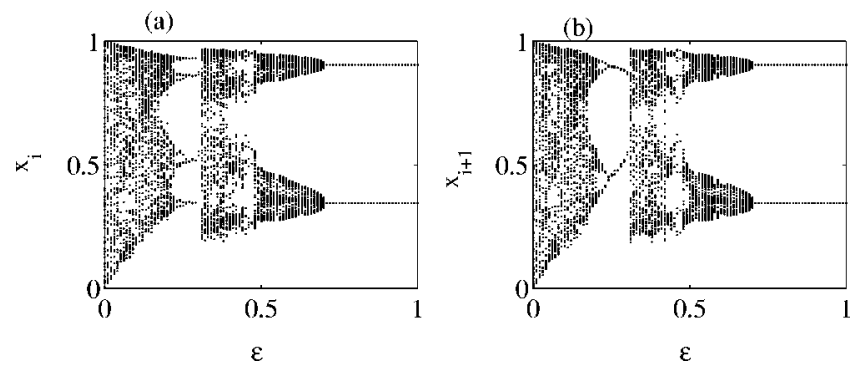

FIG. 2. Bifurcation diagram obtained numerically, integrating Eq. (3) with $a=4, k=1$, and $N=50$. We plot the values of two consecutive elements, $x_{i}$ (a) and $x_{i+1}$ (b). Notice that after a complex bifurcation scenario, the two elements of the array synchronize in a period-2 orbit.

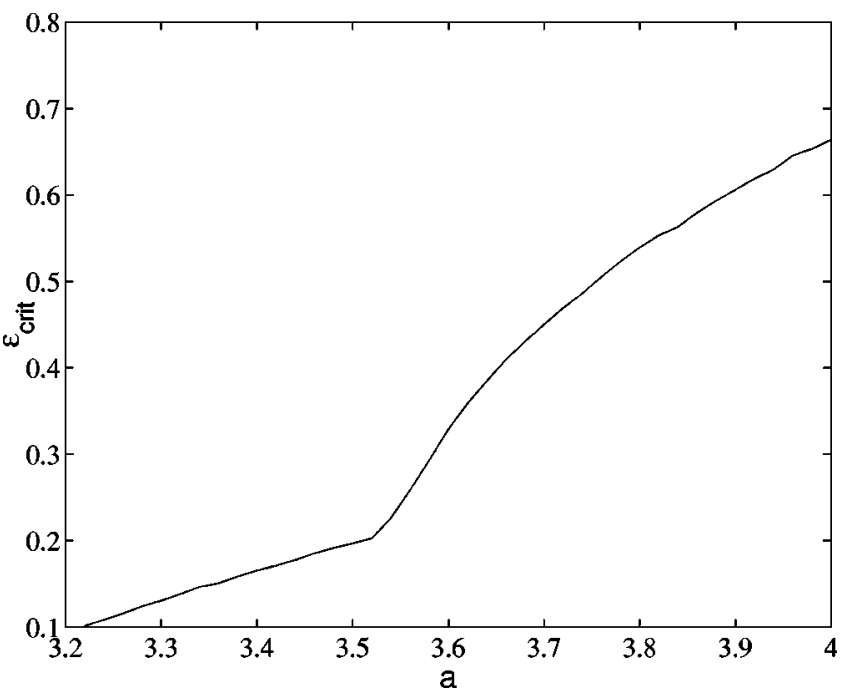

FIG. 3. Critical coupling strength above which synchronization occurs vs the nonlinear parameter $a . k=1$ and $N=100$. $\epsilon_{c r i t}$ was calculated averaging over 100 different initial conditions.

vs $\epsilon$ for $a=3.5$ and $k=1,3,5$, and 7 . Note that in a wide range of coupling strength, $\left|\lambda_{\max }\right|$ is slightly larger than 1 . In this parameter region, starting from random initial conditions there is a transient time in which the array approaches the antiphase solution; after this transient the array exhibits a complex spatiotemporal behavior. The transient time increases with $N$; as an example, Fig. 6 displays the mean value $\langle x\rangle=\sum_{i=1}^{N} x_{i}$ vs time, for four different system sizes. The study of this unexpected effect of the system size is the object of future work.

Next, we show results for the in-phase solution, which exists for $k=n P$. Figure 7 displays the bifurcation diagram for two elements of the array, and $a=3.5, k=4$, and $N$ $=50$ (for $a=3.5$, the uncoupled maps evolve in an orbit of period 4). We observe that above a critical coupling strength $\left(\epsilon_{\text {crit }} \sim 0.23\right)$, the array synchronizes in the period-4 orbit of

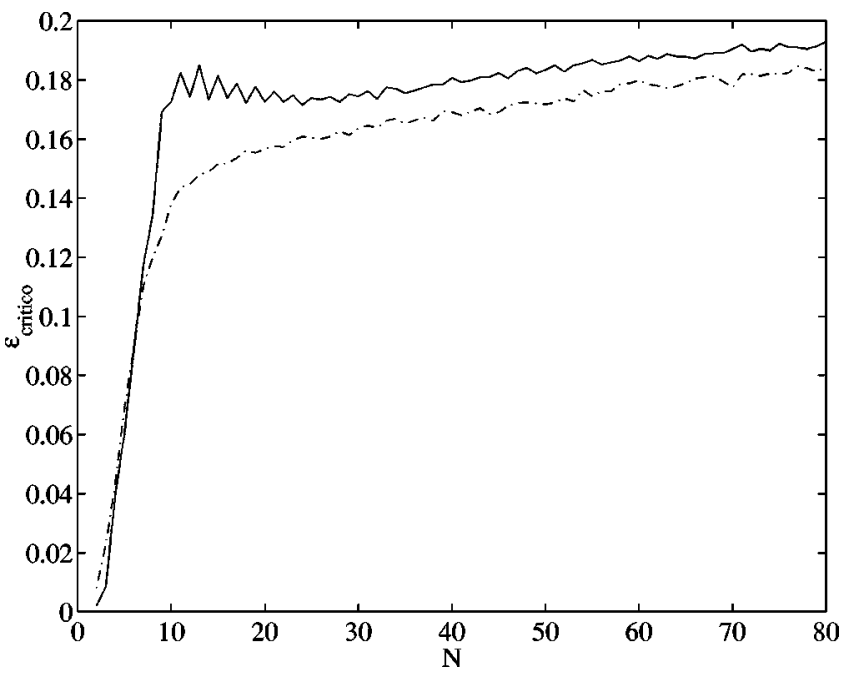

FIG. 4. Critical coupling strength above which global synchronization occurs as a function of $N$ for $a=3.5$ and $k=1$ (solid line); $a=3.5$ and $k=4$ (dashed line). 


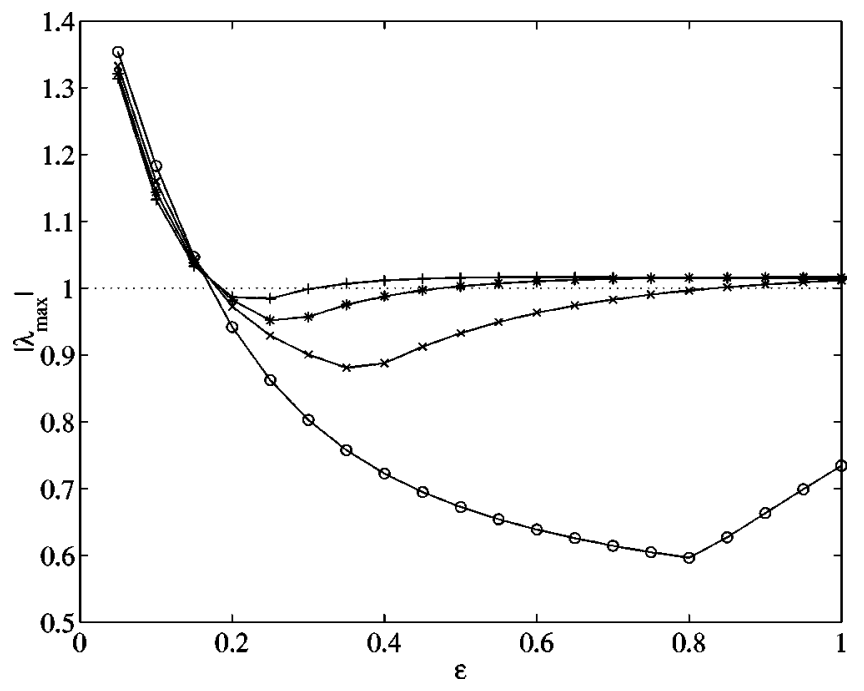

FIG. 5. Modulus of the largest eigenvalue of the matrix $A$ as a function of $\epsilon$ for the antiphase solution and $a=3.5, k=1$ (o), $k$ $=3(\mathrm{x}), k=5(*)$, and $k=7(+)$.

the uncoupled map. As in the case of the antiphase solution, for coupling strengths below $\epsilon_{\text {crit }}$, the bifurcation diagrams shown in Figs. 7(a) and 7(b) differ. This is due to the fact that the two elements belong to different clusters. The dashed line in Fig. 4 shows that $\epsilon_{\text {crit }}$ increases with increasing system size $N$.

For arbitrary values of $k, a$, and $\epsilon$, we found a rich variety of complex spatiotemporal behaviors. The characterization of the different dynamic regimes is the object of future work.

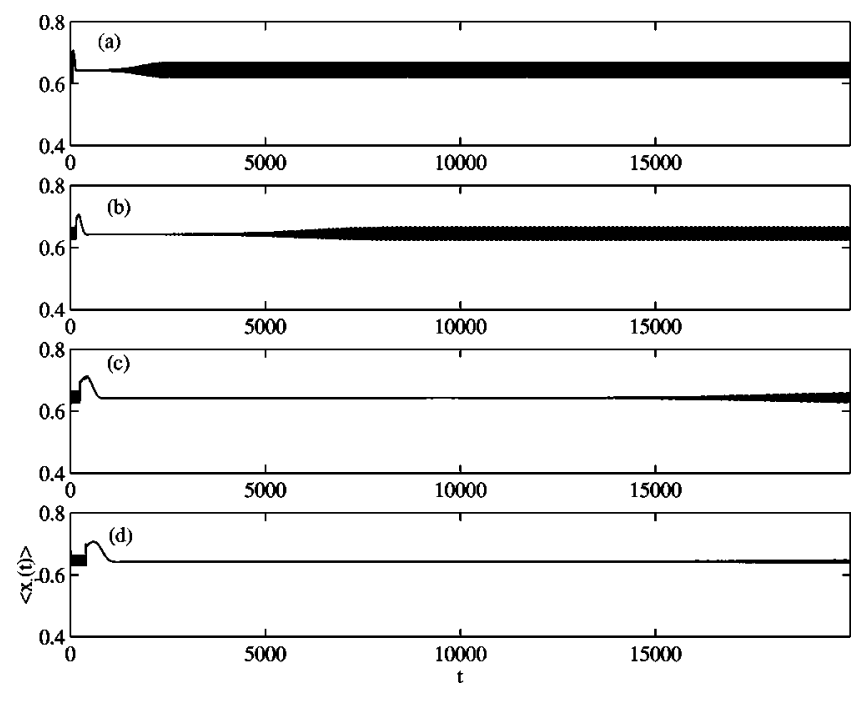

FIG. 6. Temporal evolution of the mean value $\left\langle x_{i}(t)\right\rangle$ for four different system sizes, $N=12$ (a), $N=30$ (b), $N=50$ (c), and $N$ $=80(\mathrm{~d})$. The parameters are $a=3.5, k=5$, and $\epsilon=0.6$
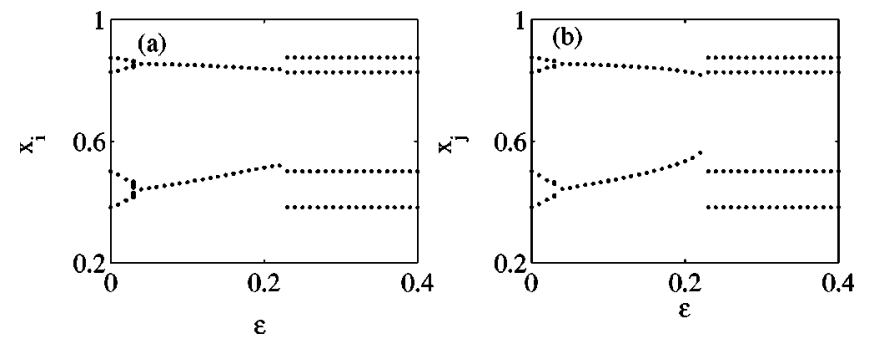

FIG. 7. Bifurcation diagram obtained numerically, integrating Eq. (3) with $a=3.5, k=4$, and $N=50$. We plot the values of two different elements of the array, $x_{i}$ (a) and $x_{j}(\mathrm{~b})$. Notice that after a period-halving bifurcation, the elements of the array synchronize in a period-4 orbit.

\section{SUMMARY AND CONCLUSIONS}

We studied the synchronization of a linear array of identical logistic maps. We consider time-delayed mutual coupling with delay times $\tau_{i j}$ that are proportional to the distance between the maps $\left(\tau_{i j}=k|i-j|\right)$. Depending on the time delays and on the coupling strength, different synchronization regimes might occur. If the coupling is weak, the array usually splits into a complex clustered structure. If the coupling is large enough, global synchronization occurs. In the globally synchronized state, each element of the array sees all other elements in its present state $\left[x_{i}(t)=x_{j}(t\right.$ $\left.\left.-\tau_{i j}\right) \forall i, j\right]$, and all the elements of the array evolve along a periodic orbit of the uncoupled maps. The spatial correlation along the array is either periodic or homogeneous depending on $k$. If $k$ is odd, the array synchronizes in antiphase, such that the state at time $t$ of two consecutive elements is $x_{i}(t)$ $=x_{0}(t), x_{i+1}(t)=x_{0}(t+P / 2) \quad\left[\right.$ where $x_{0}(t)$ is a particular realization of the orbit of period $P$, used as a reference]. If $k=n P$, the array synchronizes in phase, such that the state at time $t$ is $x_{i}(t)=x_{0}(t) \forall i$. For parameter values such that the uncoupled maps are chaotic, mutual delayed coupling suppresses chaos, rendering the evolution of the elements of the array periodic in time. Thus, an important consequence of our analysis is that delayed coupling might allow controlling an assembly of chaotic maps by rending an unstable periodic orbit of the uncoupled maps, stable. In addition, the antiphase synchronization regime found here might be of interest in the context of population models [15-17] where an increase of the connectivity among isolated populations leads to in-phase synchronization of local population oscillations and thereby increases the danger of global extinction. Our results suggest that if a distant-dependent delay is taken into account, under appropriate conditions an increase of the connectivity might lead to coherent antiphase oscillations of the local populations, thus avoiding the danger of global extinction.
[1] A.S. Pikovsky, M.G. Rosenblum, and J. Kurths, Synchronization-A Universal Concept in Nonlinear Sciences (Cambridge University Press, Cambridge, 2001).
[2] S. Boccaletti, J. Kurths, G. Osipov, D.L. Valladares, and C.S. Zhou, Phys. Rep. 366, 1 (2002).

[3] K. Kaneko, Phys. Rev. Lett. 63, 219 (1989). 
[4] K. Kaneko, Physica D 41, 137 (1990).

[5] A. Pikovsky, O. Popovych, and Y. Maistrenko, Phys. Rev. Lett. 87, 044102 (2001).

[6] O. Popovych, A. Pikovsky, and Yu. Maistrenko, Physica D 168-169, 106 (2002).

[7] J. Garcia-Ojalvo, J. Casademont, C.R. Mirasso, M.C. Torrent, and J.M. Sancho, Int. J. Bifurcation Chaos Appl. Sci. Eng. 9, 2225 (1999).

[8] G. Kozyreff, A.G. Vladimirov, and P. Mandel, Phys. Rev. Lett. 85, 3809 (2000).

[9] Y. Jiang, Phys. Lett. A 267, 342 (2000).

[10] P. Parmananda, M. Hildebrand, and M. Eiswirth, Phys. Rev. E
56, 239 (1997).

[11] P.M. Gade, Phys. Rev. E 57, 7309 (1998).

[12] D.H. Zanette, Phys. Rev. E 62, 3167 (2000).

[13] S.O. Jeong, T.W. Ko, and H.T. Moon, Phys. Rev. Lett. 89, 154104 (2002).

[14] C. Masoller, A.C. Martí, and D. Zanette (unpublished).

[15] A. Hastings, Ecology 74, 1362 (1993).

[16] B. Blasius, A. Huppert, and L. Stone, Nature (London) 399, 354 (1999).

[17] D.J.D. Earn, S.A. Levin, and P. Rohani, Science (Washington, DC, U.S.) 290, 1360 (2000). 